\title{
CALCULI OF INDIVIDUALS AND SOME EXTENSIONS: AN OVERVIEW ${ }^{*}$
}

\author{
KARL-GEORG NIEBERGALL \\ Humboldt University Berlin
}

\section{INTRODUCTION}

Calculi of individuals are usually regarded as paradigmatic examples of nominalistic theories. ${ }^{1}$ This view is fine with me. ${ }^{2}$ Nonetheless, it may be asked: what do or should we understand by "calculus of individuals" and "nominalistic theory"? Now, although there are clear cases of theories which are accepted as nominalistic or calculi of individuals - or fail to be so - I admit that we have no general convincing explications of these predicates at our disposal. In the case of "nominalistic theories", I guess this is something we have to live with; but that may be nothing to worry about (see Niebergall 2005 and 2007 for more on this topic, and also footnote 5). When it comes to calculi of individuals, however, the situation is different, and it may be better. As a starting point, let me repeat an explication of "calculus of individuals" from Niebergall 2007.

Let $\mathrm{L}^{1}[\mathrm{o}]$ be the $1^{\text {st }}$ order language with the 2-place predicate "o" read "overlaps" - as its sole non-logical primitive expression. Consider the theory $\mathrm{CI}$ in $\mathrm{L}^{1}[\mathrm{o}]$ axiomatized as follows: ${ }^{3}$

$$
\begin{array}{ll}
\text { O } & \forall x y(\exists z(z \Pi x \wedge z \Pi y) \leftrightarrow x \text { o } y), \\
\text { SUM } & \forall x y \exists z \forall u(z \circ u \leftrightarrow x \text { o } u \vee y \circ u), \\
\text { NEG } & \forall x(\exists y \forall u(u \Pi y \leftrightarrow \neg u \text { o } x) \leftrightarrow \neg \forall w w \circ x) .
\end{array}
$$

\footnotetext{
* This paper was presented under the title "Mereologische Theorien" at the 31 st International Wittgenstein Symposium 2008. I would like to thank the scientific directors, Alexander Hieke and Hannes Leitgeb, and the Österreichische Ludwig Wittgenstein Gesellschaft for the invitation, and the participants of the talk for comments.

${ }^{1}$ For Goodman (1951), the calculus of individuals was a specific theory in $\mathrm{L}^{1}[\mathrm{o}]$; it is called "CI + FUS" ${ }^{1}$ " below. In the meantime, "calculus of individuals" has become a predicate which is now ascribed to many theories.

${ }^{2}$ Let me add, however, that even if the first statement of this paper is beyond dispute for $1^{\text {st }}$ order languages and theories, it might be worthwhile to rethink it for $2^{\text {nd }}$ order ones.

3 “ $y$ is part of $x "$ is defined as follows: $y \Pi x: \leftrightarrow \forall u(u$ o $y \rightarrow u$ o $x)$.
} 
Let's then define:

(D1) $T$ is a calculus of individuals $: \Leftrightarrow T$ is formulated in $\mathrm{L}^{1}[\mathrm{o}] \wedge \mathrm{CI} \subseteq T$

Being formulated in $\mathrm{L}^{1}[\mathrm{o}]$ is certainly not sufficient for a theory $T$ to be called a "calculus of individuals": "o" might be axiomatized in a way which does not conform to its preferred paraphrase "overlaps". The addiion of " $\mathrm{CI} \subseteq T$ " is supposed to exclude such unintended readings (cf. Niebergall 2007). Are there proper extensions $T$ of CI formulated in $\mathrm{L}^{1}$ [o] which should not be regarded as calculi of individuals (see section 2.1 for some of them)? This is difficult to say. For on the one side, I doubt that there are such $T$ which have about the same degree of plausibility as CI, given the intended readings "overlaps" and "part of" of "o" and " $\Pi$ ": only CI is well motivated. Yet, if no sentence independent of CI can be determined as being correct (under the intended reading), none can be pinned down as incorrect. But on the other side, whatever the intended reading of $\mathrm{L}^{1}[\mathrm{o}]$ is, if $\varphi$ is a sentence of $\mathrm{L}^{1}[\mathrm{o}]$, either it or its negation must be true under that reading. Nonetheless, our linguistic intuitions on their own are just not determined enough for us to answer which one.

Actually, whereas we may want to determine a unique correct theory with a unique model (up to isomorphism) when doing number theory (which sadly does no work nicely), calculi of individuals are used with a different aim: they should be applicable in many situations, that is, for many quite diverse relational systems which only have some structural similarities. In that situation, I think that the appropriate general methodic approach is to attempt to obtain results about all the theories from $\mathrm{L}^{1}$ [o] which extend CI. For with this method, we get those results also for the "real" calculi of individuals (in $\mathrm{L}^{1}[\mathrm{o}]$ ), whatever they may be.

Coming back to (D1), we have to deal with a further possibility: There could be theories $T$ formulated in extensions $L+$ of $L^{1}[o]$ that deserve to be called "calculi of individuals".

I would certainly not regard all such $T$ as calculi of individuals. As an example, take an $\mathrm{L}+$ which results from $\mathrm{L}^{1}[\mathrm{o}]$ by the addition of the 2place predicate " $\in$ ", and let $T$ be $\mathrm{CI}+\mathrm{ZF}$ (where the ZF-component is stated only in that part of $\mathrm{L}+$ which is built over " $\in$ "). 
But then, consider these types of extensions $\mathrm{L}+$ of $\mathrm{L}^{1}[\mathrm{o}]$ :

$-\mathrm{L}+$ is $1^{\text {st }}$ order, but contains additional predicates or functions signs, such as: "is with", "has the same size as", "matches", "is a quale" (see Goodman 1951, Breitkopf 1978); "is connected with", "lies in the interior of" (i.e., topological vocabulary); "lies between", "is congruent with" (i.e., geometrical vocabulary); "is next to" (see Lewis 1970); "contains fewer points than" (Field 1980), "is longer than", "has more bits than"; "is finite".

$-\mathrm{L}+$ is a $2^{\text {nd }}$ order language which results from $\mathrm{L}^{1}[\mathrm{o}]$ by the addition of monadic $2^{\text {nd }}$ order variables (see Field 1980, Lewis 1991). ${ }^{4}$

And take theories $T$ in these languages which fix the use of the new expressions in a way appropriate to the readings just given (thus, they are supposed to extend CI).

It has been suggested repeatedly that these $\mathrm{L}+$ and $T$ are nominalistically admissible (see, e.g., Lewis 1970, Field 1980; for $2^{\text {nd }}$ order languages in particular, see Leonard/Goodman 1940, Field 1980, Lewis 1991); and the $T$ have also been classified as calculi of individuals (see Clarke 1981, 1985; for both claims, see Goodman 1951, Breitkopf 1978). Moreover, for $1^{\text {st }}$ order theories this has usually been done without much further ado. A reason for the latter assessments could be that in these cases, the relata of the newly introduced relations are most naturally viewed as concrete objects, or individuals, or particulars. ${ }^{5}$ Of course, all of this is far from providing a general explicans or criterion for " $T$ is a calculus of individuals". Since, however, it seems that serious doubts have not been raised as to the rightfulness of the above classifications, I accept them here ${ }^{6}$ and call those $T$ just mentioned " $\mathrm{L}+$ calculi of individuals".

In this text, I will, adhering to the general methodic approach just sketched, first present a sort of a classification theorem and some further relevant metalogical results for almost all extensions of CI which are for-

4 One may also think about the addition of "unusual" quantifiers (i.e., quantifiers which are not $1^{\text {st }}$ order definable) to $1^{\text {st }}$ order languages. In the context of a nominalistic program, this has been suggested by Field 1980.

5 Lewis (1970) is an exception, citing Goodman 1951 and 1958. But the definitions of „,nominalistic system“ Goodman puts forward there is not precise; moreover, it may be interpreted as implying that CI + ZF is nominalistic (see Niebergall 2005 for more on this).

${ }^{6}$ As far as I know, "calculus of individuals" is in distinction to, e.g., "nominalism" and "individual", a term of art. Therefore, it is hard to find criteria for its proper use which are independent from what the philosophers and logicians working on calculi of individuals have stipulated. 
mulated in $\mathrm{L}^{1}[\mathrm{o}]$. When it comes to $\mathrm{L}+$ calculi of individuals, however, the domain of admissible languages and theories (even for a specific choice of $\mathrm{L}+$ ) is much too wide and open for similar metatheorems to hold. Here, I merely state a general dilemma for such theories and give two examples for it: one is taken from mereotopology, the other is concerned with infinity. In the final section, $2^{\text {nd }}$ order variants of calculi of individuals are addressed. For reasons of space, much will be carried out only sketchily. In particular, theorems will be stated without proofs.

\section{CAlCuli OF INDividuals IN L ${ }^{1}[\mathrm{o}]$}

Let's start with identity. I prefer to treat "=" as a "logical sign" which is axiomatized via " $x=y \leftrightarrow \forall u(u$ o $y \leftrightarrow u$ o $x)$ ", reflexivity and substitutivity (a schema that is stated for $\mathrm{L}^{1}[\mathrm{o}]$ first, but which I assume to be extended to whatever language $\mathrm{L}+$ is taken into account). ${ }^{7}$

\subsection{Optional axioms and theories for "o"}

Then we also have several options for axioms which are specific for "o". First some abbreviations:

$$
\begin{aligned}
& \operatorname{At}(y): \leftrightarrow \forall z(z \Pi x \rightarrow z=x) \text { (" } y \text { is an atom") } \\
& \left.y \Pi^{-} x: \leftrightarrow y \Pi x \wedge \neg(x \Pi y) \text { (" } y \text { is a proper part of } x "\right) \\
& \exists_{>_{n}} x \operatorname{At}(x): \leftrightarrow \exists x_{0} \ldots x_{n}\left(\operatorname{At}\left(x_{0}\right) \wedge \ldots \wedge \operatorname{At}\left(x_{n}\right) \wedge x_{0} \neq x_{1} \wedge \ldots \wedge x_{n-1} \neq x_{n}\right) \\
& \quad \text { ("there are more than } n \text { atoms") } \\
& \exists_{n+1} x \operatorname{At}(x): \leftrightarrow \exists_{>_{n}} x \operatorname{At}(x) \wedge \neg \exists_{>(n+1)} x \operatorname{At}(x) \text { ("there are } n+1 \text { atoms") }
\end{aligned}
$$

Now the axioms:

$\begin{array}{ll}\text { AT } & \forall x \exists y(y \Pi x \wedge \operatorname{At}(y)) \\ \text { AF } & \forall x \exists y y \Pi^{-} x \\ \text { HYPEXT } & \forall x y[\forall z(\operatorname{At}(z) \wedge z \Pi y \leftrightarrow \operatorname{At}(z) \wedge z \Pi x) \rightarrow x=y] \\ \text { DE } & \forall x y\left(y \Pi^{-} x \rightarrow \exists z\left(y \Pi^{-} z \wedge z \Pi^{-} x\right)\right) \\ \text { PROD } & \forall x y(x \circ y \rightarrow \exists z \forall u(u \Pi z \leftrightarrow u \Pi x \wedge u \Pi y))\end{array}$

7 "=" could also be defined in $\mathrm{L}^{1}[\mathrm{o}]: x=y: \leftrightarrow \forall u(u$ o $y \leftrightarrow u$ o $x)$. But here, too, the adjustment has to be taken care of. 
There are also two axiom-schemata: the fusion schema $\mathrm{FUS}^{1}$ (with variants; see, e.g., Eberle 1970) and the nucleus schema (see Goodman 1951, Breitkopf 1978). Since the latter follows from CI $+\mathrm{FUS}^{1}$, I only deal with FUS ${ }^{1}$. Thus, let $\varphi(x)$ be a formula of $\mathrm{L}^{1}[\mathrm{o}]$ (possibly containing further free variables). Then set

$$
\operatorname{FUS}_{\varphi}^{1}:=\lceil\exists x \varphi(x) \rightarrow \exists z \forall y(z \text { o } y \leftrightarrow \exists x(\varphi(x) \wedge x \text { o } y))\rceil
$$

and let $\mathrm{FUS}^{1}$ be the set of all the formulas $\mathrm{FUS}_{\varphi}^{1}$ (with $\varphi$ in $\mathrm{L}^{1}[\mathrm{o}]$ ).

The intended reading of these sentences is obvious: AT asserts that each object has an atomic part, AF that each object has a proper part. HYPEXT says that objects are determined by their atomic parts (see Goodman 1958, Yoes 1967), DE expresses density (and possibly also infinity), PROD that meets of overlapping objects exist (note that we do not have a null object). Finally, FUS $^{1}$ is an infinitary version of SUM.

Are there further natural axioms in $\mathrm{L}^{1}[\mathrm{o}]$ which are in harmony with CI? There could well be: it depends on the reader's imagination to find them. But in a specific sense, the answer is (almost): No; see section 2.4. Moreover, some of the above axioms are superfluous.

Lemma 1: $\mathrm{CI} F \mathrm{AT} \leftrightarrow \mathrm{HYPEXT}, \neg \mathrm{AF} \leftrightarrow \exists x \mathrm{At}(x), \mathrm{AF} \rightarrow \mathrm{DE}$, $\exists y \forall x x \Pi y$, PROD.

Given the above implications between the optional additional axioms, the following are the theories in $\mathrm{L}^{1}[\mathrm{o}]$ which suggest themselves as extensions of CI.

First there are the extensions of CI by AT, AF and their negations:

$\mathrm{ACI}:=\mathrm{CI}+\mathrm{AT}$ (for "atomic calculus of individuals"),

$\mathrm{FCI}:=\mathrm{CI}+\mathrm{AF}$ (for "atomfree calculus of individuals"),

$\mathrm{MCI}:=\mathrm{CI}+\neg \mathrm{AT}+\neg \mathrm{AF}$ (for "mixed calculus of individuals").

Second there are extensions of those in which the number of the atoms is addressed:

$$
\begin{aligned}
& \mathrm{ACI}_{>n}:=\mathrm{ACI}+\left\{\exists_{>_{n}} x \operatorname{At}(x)\right\}, \\
& \mathrm{ACI}_{n+1}:=\mathrm{ACI}+\left\{\exists_{n+1} x \operatorname{At}(x)\right\}, \\
& \mathrm{ACI}_{\omega}:=\mathrm{ACI}+\left\{\exists_{>n} x \operatorname{At}(x) \mid n \in \omega\right\}, \\
& \mathrm{MCI}_{>_{n}}:=\mathrm{MCI}+\left\{\exists_{>_{n}} x \operatorname{At}(x)\right\},
\end{aligned}
$$




$$
\begin{aligned}
& \operatorname{MCI}_{n+1}:=\mathrm{MCI}+\exists_{n+1} x \operatorname{At}(x), \\
& \operatorname{MCI}_{\omega}:=\mathrm{MCI}+\left\{\exists_{>_{n}} x \operatorname{At}(x) \mid n \in \omega\right\} .
\end{aligned}
$$

Of course, in each case instances of FUS $^{1}$ could be added as further axioms. $^{8}$

\subsection{The general methodic approach and metalogical considerations}

Given the general methodic approach from section 1, the best one could hope for here would be a complete grasp of all the consistent extensions $T$ of $\mathrm{CI}$ in $\mathrm{L}^{1}[\mathrm{o}]$. For a start, this includes the investigation of what may be called ,absolute properties“ of such $T$. Thus, we should ask

(F1) Which $T$ are consistent, maximal consistent, decidable, $\kappa$-categorical etc.

But we should also be interested in ,relational properties" of these $T$, i.e., in intertheoretic relations between them, and in relations between them and established other theories. I believe that for these aims, one should address:

(F2) Which arithmetic and set theories are relatively interpretable in extensions of CI?

(F3) What is the relation between the extensions of CI with respect to relative interpretability?

Whereas almost nothing has been published on (F2) and (F3), some partial results concerning (F1) can be found in the relevant literature:

(i) ACI is decidable.

(ii) Each $\mathrm{ACI}_{n+1}$ is categorical, maximal consistent and decidable.

\footnotetext{
${ }^{8} \mathrm{ACI}$, the $\mathrm{ACI}_{n+1}$ 's and $\mathrm{ACI}_{\omega}$ are the theories treated in Hodges/Lewis 1968. The theory presented in Goodman 1951 amounts to CI + FUS ${ }^{1}$ (see also Breitkopf 1978, Ridder 2002). For the other theories, see Hendry 1982.

${ }^{9}$ A relative interpretation from theory $S$ in theory $T$ is a map from the set of formulas of $\mathrm{L}[S]$ to the set of formulas of $\mathrm{L}[T]$ which preserves quantificational structure (apart from relativizing quantifiers) and maps $S$ into $T$; see Tarski et.al. 1953 and Feferman 1960. For a defense of the claim that relative interpretability provides a good explicans for "reducibility", see Niebergall 2000 and 2005.
} 
(iii) $\mathrm{ACI}_{\omega}$ is maximal consistent and decidable (of course, it cannot be categorical).

(iv) $\mathrm{ACI}_{\omega}$ is not $\boldsymbol{\aleph}_{0}$-categorical.

(v) $\mathrm{FCI}+\mathrm{FUS}^{1}$ is $\boldsymbol{\aleph}_{0}$-categorical, maximal consistent and decidable.

(vi) Each $\mathrm{MCI}_{n+1}+\mathrm{FUS}^{1}$ is $\boldsymbol{\aleph}_{0}$-categorical, maximal consistent and decidable.

(vii) The maximal consistent extensions of ACI are exactly the $\mathrm{ACI}_{n+1}$ and $\mathrm{ACI}_{\omega}$.

For (i), (ii), (iii) and (vii) see Hodges/Lewis 1968 (they established (ii) via a normal form theorem; but a model-theoretic proof is easier and obvious). For (iv) see Hellman 1969. For (v) and (vi), see Hendry 1982.

Section 2.4 contains a report about strengthenings of these metatheorems. In particular, I present a comprehensive list of the maximal consistent extensions of CI + FUS $^{1}{ }_{\text {AT. }}$. Here, FUS $^{1}{ }_{\text {AT }}$ abbreviates " $\exists x \operatorname{At}(x) \rightarrow \exists z \forall y$ $(z \circ y \leftrightarrow \exists x(\operatorname{At}(x) \wedge x \circ y))$ ". Furthermore, in section 2.5 there are results concerning (F2) and (F3). But let's start with Boolean algebras, which turn out to be very useful.

\subsection{Mereological and Boolean Algebras}

For structures in which $\mathrm{L}^{1}[\mathrm{o}]$ can be evaluated, I usually write $\left\langle M, \mathrm{o}^{M}\right\rangle$ (=: $\mathscr{C}$; with $M \neq \varnothing$ and $\mathrm{o}^{M} \subseteq M^{2}$ ). Those of them which satisfy CI I call "mereological algebras". A Boolean algebra $\mathscr{B}$, which is a structure of the form $\left\langle B, \Pi^{B}, \sqcup^{B},-{ }^{B}, 0^{B}, 1^{B}\right\rangle$, may also be viewed as a model of a theory BA which is stated in the corresponding equational $1^{\text {st }}$ order language L[BA] (see Halmos 1963).

Despite their different signatures, it is quite easy to turn a Boolean algebras into a mereological algebra and vice versa (and it is well known how to do it). For my purposes, it suffices to explain how to obtain a mereological algebra $\mathscr{B}$ from a Boolean algebra $\mathscr{B}$.

$$
\mathscr{B}=\left\langle B^{-}, \mathrm{o}^{B}\right\rangle \text {, with } B^{-}:=B \backslash\left\{0^{B}\right\} \text { and for } a, b \text { in } B^{-}, a \mathrm{o}^{B-} b: \Leftrightarrow a \Pi^{B} b \neq 0^{B} \text {. }
$$

This correspondence induces a relative interpretation $\mathscr{T}$ from CI in BA which is faithful (i.e., nontheorems of CI are mapped to nontheorems of BA). $\mathscr{T}$ being a recursive function, we therefore get at once the first 
main result from the decidability of BA (for a detailed sketch of the proof, see Niebergall 2007):

Theorem 1: CI is decidable.

Boolean algebras also provide for models of the theories from section 2.1:

Set $\mathscr{B}_{n+1}=\langle\wp(\{0, \ldots, n\}), \cap, \cup, \backslash, \varnothing,\{0, \ldots, n\}\rangle$;

then $\mathscr{B}_{n+1}{ }^{-} \mid=\mathrm{ACI}_{n+1}+\mathrm{FUS}^{1}$.

Set $\mathscr{R}_{0}=\langle\wp(I N), \cap, \cup, \backslash, \varnothing, I N\rangle$; then $\mathscr{R}_{\omega}^{-} \mid=\mathrm{ACI}_{\omega}+\mathrm{FUS}^{1}$.

Let $\mathrm{RO}(I R)$ be the set of regular open sets of $I R$ (given the usual topology on $I R$ ) and set $\mathscr{B}_{R}=\langle\mathrm{RO}(I R), \cap, \cup, \backslash, \varnothing, I R\rangle$; then $\mathrm{B}_{\mathrm{R}}{ }^{-}=\mathrm{FCI}+\mathrm{FUS}^{1}$.

Consider $\mathscr{P}_{n+1} \times \mathscr{P}_{R}$ (the product of $\mathscr{P}_{n+1}$ with $\mathscr{P}_{R}$, explained in the natural way); then $\left(\mathscr{B}_{n+1} \times \mathscr{B}_{R}\right)^{-} \mid=\mathrm{MCI}_{n+1}+\mathrm{FUS}^{1}$.

Consider $\mathscr{B}_{\omega} \times \mathscr{B}_{R}$; then $\left(\mathscr{B}_{\omega} \times \mathscr{B}_{R}\right)^{-} \mid=\mathrm{MCI}_{\omega}+\mathrm{FUS}^{1}$.

\subsection{Absolute metalogical results: (F1)}

First, we have some slight amendments of the results mentioned in section 2.2:

(v) + FCI is $\aleph_{0}$-categorical, maximal consistent and decidable.

(vi) + Each $\mathrm{MCI}_{\mathrm{n}+1}$ is $\boldsymbol{\aleph}_{0}$-categorical, whence maximal consistent and decidable.

Then there is the second main new result:

Theorem 2: $\mathrm{MCI}_{\omega}+\mathrm{FUS}^{1}{ }_{\mathrm{AT}}$ is maximal consistent and decidable, but not $\aleph_{0}$-categorical.

As a roundup, there is what I have called a classification theorem:

Theorem 3: The maximal consistent extensions of $\mathrm{CI}+\mathrm{FUS}_{\mathrm{AT}}{ }_{\mathrm{T}}$ in $\mathrm{L}^{1}[\mathrm{o}]$ are exactly the $\mathrm{ACI}_{\mathrm{n}+1}$ and $\mathrm{MCI}_{\mathrm{n}+1}, \mathrm{ACI}_{\omega}, \mathrm{FCI}$ and $\mathrm{MCI}_{\omega}+$ FUS $^{1}$ AT. 
This has surprising and nice consequences, such as:

\section{Corollary 1:}

(i) $\mathrm{ACI}-\mathrm{FUS}^{1}$, and CI $+\mathrm{FUS}^{1}{ }_{\mathrm{AT}}+\mathrm{FUS}^{1}$.

(ii) $\quad \mathrm{ACI}=T h$ (the class of finite mereological algebras)

(which is decidable). ${ }^{10}$

\subsection{Relative interpretability: (F2) and (F3)}

Calculi of individuals should be widely applicable; but they are not supposed to contain only logical truths. In fact, it has been repeatedly suggested that they can play the role of set theories while at the same time avoiding the unpleasant ontological commitments of the latter. This lends a particular importance to (F2). Now, Theorem 1 quite directly yields a strong answer to that question:

Theorem 4: No consistent extension of CI (in $\mathrm{L}^{1}[\mathrm{o}]$ ) interprets Q. ${ }^{11}$

The consequences of this metatheorem for a nominalistic reduction program should be taken seriously; but I think there are not disastrous. There are other theories which come to the nominalist's rescue (see Niebergall 2005 for a suggestion).

Let me finally come to (F3). Here is a list of the central (non-) interpretability results I am aware of. In what follows, " $S \leqslant T$ ' is supposed to stand for " $S$ is relatively interpretable in $T$ ".

Theorem 5: For arbitrary $k, n \in I N$,

(i) $\neg\left(\mathrm{ACI}_{n+1} \leqslant \mathrm{ACI}_{k+1}\right)$, if $n \neq k$,

$\neg\left(\mathrm{ACI}_{\omega} \leqslant \mathrm{ACI}_{n+1}\right), \neg\left(\mathrm{ACI}_{n+1} \preccurlyeq \mathrm{ACI}_{\omega}\right)$,

$\neg\left(\mathrm{FCI} \preccurlyeq \mathrm{ACI}_{n+1}\right)$,

$\neg\left(\mathrm{FCI} \preccurlyeq \mathrm{ACI}_{\omega}\right), \neg\left(\mathrm{ACI}_{\omega} \leqslant \mathrm{FCI}\right)$,

(ii) $\mathrm{ACI} \preccurlyeq \mathrm{MCI}+\mathrm{FUS}_{\mathrm{AT}}^{1}, \mathrm{ACI}_{n+1} \preccurlyeq \mathrm{MCI}_{n+1}$, $\mathrm{ACI}_{\omega} \leqslant \mathrm{MCI}_{\omega}+\mathrm{FUS}_{\mathrm{AT}}^{1}$,

\footnotetext{
${ }^{10}$ Proofs can be found in the unpublished manuscript Niebergall 2009b; see also Niebergall 2007. The proofs of (vi)+ and Theorem 2 use a specific back-and-forth-construction, plus results mentioned in section 2.5.

${ }^{11}$ Q, i.e., Robinson-Arithmetic, is a weak subtheory of PA; cf. Tarski et.al. 1953. For a transfer of Theorem 4 to the domain of set theories and further refinements, see Niebergall 2007.
} 
(iii) $\neg\left(\mathrm{MCI} \leqslant \mathrm{ACI}_{n+1}\right), \neg\left(\mathrm{MCI} \leqslant \mathrm{ACI}_{\omega}\right), \neg\left(\mathrm{ACI}_{\omega} \leqslant \mathrm{MCI}_{n+1}\right)$,

(iv) $\mathrm{FCI} \preccurlyeq \mathrm{MCI}+\mathrm{FUS}^{1}{ }_{\mathrm{AT}}$.

Some of these claims follow by applying general metatheorems about relative interpretability. (ii) and (iv) rest on specifically chosen translations $\mathscr{T}_{\mathrm{A}}$ and $\mathscr{T}_{\mathrm{F}}$. In both cases, " $x$ o $y$ " is mapped to itself. Moreover (employing quasi-quotation),

$$
\begin{aligned}
& \mathscr{T}([\forall x \varphi\rceil)=\left\lceil\forall x\left(\delta_{\mathrm{A}}(x) \rightarrow \mathscr{T}(\varphi)\right)\right\rceil \text { and } \\
& \mathscr{T}(\forall x \varphi\rceil)=\left\lceil\forall x\left(\delta_{\mathrm{F}}(x) \rightarrow \mathscr{T}(\varphi)\right)\right),
\end{aligned}
$$

where $\delta_{\mathrm{A}}(x)=\lceil\forall y(y \Pi x \rightarrow \exists z(z \Pi y \wedge \operatorname{At}(z)))\rceil$ and $\delta_{\mathrm{F}}(x)=\lceil\forall y(y \Pi x \rightarrow \neg \operatorname{At}(y))\rceil$.

\section{CALCULI OF INDIVIDUALS IN EXTENDED $1^{\text {ST }}$ ORDER LANGUAGES}

\subsection{The WS-dilemma}

Why have $1^{\text {st }}$ order extensions $\mathrm{L}+$ of $\mathrm{L}^{1}[\mathrm{o}]$ and $\mathrm{L}+$ calculi of individuals $T$ been introduced in the first place? I presume that from a systematic point of view, the answer is: to have languages that enrich $\mathrm{L}^{1}[\mathrm{o}]$ and theories that strengthen the calculi of individuals as defined by (D1), yet nevertheless preserve the salient features of the latter. Now, I think that those salient features include above all freedom from ontological commitment to abstract objects or universals. Actually, I find it somewhat difficult to understand how calculi of individuals could be interesting when they are not viewed as contributions to a nominalistic reduction program. To this, one may answer with recourse to the idea of resource boundedness: Calculi of individuals are proof theoretically weak, but just strong enough for what they are supposed to deliver. But why could not also, e.g., weak set theories be suitable for this role?

Be this as it may - I think that these considerations point to what may be a dilemma for L+ calculi of individuals: the WS-dilemma (for "wide-strong-dilemma"), as I will call it.

On the one side, as I understand the above mentioned "to have languages that enrich $\mathrm{L}^{1}[\mathrm{o}]$ and theories that strengthen the calculi of individuals as defined by (D1)", it should imply at least that if $T$ is a calculus of individuals in $\mathrm{L}^{1}[\mathrm{o}]$, an extension $S$ of $T$ in $\mathrm{L}+$ should not merely be a definitional extension of $T$. With the abbreviation 


$$
S \mid \mathrm{L}^{1}[\mathrm{o}]:=\left\{\varphi \mid \varphi \text { is a } \mathrm{L}^{1}[\mathrm{o}] \text {-sentence } \wedge S \vdash \varphi\right\},
$$

this can be stated as follows (for "possible definition", see Tarski et.al. 1953):

(S) Not: for each consistent $\mathrm{L}+$ calculus of individuals $S$, there are possible definitions $D$ in $\mathrm{L}^{1}[\mathrm{o}]$ for the new vocabulary from $\mathrm{L}+$ such that $S$ is a subtheory of $S \mid \mathrm{L}^{1}[\mathrm{o}]$ extended by $D .^{12}$

On the other side, just as for calculi of individuals in $\mathrm{L}^{1}[\mathrm{o}]$, there is no unique intended $\mathrm{L}+$ calculus of individuals - at least if $\mathrm{L}+$ is one of the extensions of $\mathrm{L}^{1}[\mathrm{o}]$ mentioned in the introduction. That is, if one wants to add only evident or at least rather plausible new axioms formulated in $\mathrm{L}+$ to a calculus of individuals $T$ in $\mathrm{L}^{1}[\mathrm{o}]$, one will most probably obtain rather weak extensions of $T$. And since for such a $T$, only $\mathrm{CI}$ is a choice which is beyond dispute, we get in particular:

(W) If $S$ is a consistent $\mathrm{L}+$ calculus of individuals, $S \mid \mathrm{L}^{1}[\mathrm{o}]$ is not maximal consistent (in $\mathrm{L}^{1}[\mathrm{o}]$ ).

(S) and (W) certainly do not contradict each other. But it may be that, in practice, they are not easily realized simultaneously. Let me present examples for that claim in the following two subsections.

\subsection{Mereotopology}

Topology is a mathematical discipline dealing with topological spaces and (structure preserving) mappings between them. In that approach, " $x$ is a topological space" is defined purely set theoretically: no topological axioms are given. Now Kuratowski has found a definiens for "topological space" which does not use " $\in$ ", but only " $\subseteq$ " in an essential way. This can therefore easily be turned into axioms. Thus, let's extend $\mathrm{L}^{1}[\mathrm{o}]$ by a function sign " $C$ " (read: "closure of") to $\mathrm{L}^{1}[\mathrm{o}, C]$ and consider the new axioms:

$$
\text { (AxC) } \forall x x \Pi C x, \forall x y(x \Pi y \rightarrow C x \Pi C y), \forall x C x=C C x
$$

\footnotetext{
${ }^{12}$ This way of making the basic intuition precise is just a first suggestion. It may be weakened, e.g., by mentioning relative interpretations instead of definitions, but also strengthened. Actually, the latter takes place in Lemmata 2 and 4.
} 
Here we have a version of mereotopology. It can be found in Grzegorczyk 1951 (in principle), but surprisingly has not been very successful: for alternative mereotopological vocabularies see Clarke 1981, 1985 (adding "is connected to"), Kleinknecht 1992 and Smith 1996 (adding "is an interior part of") and perhaps Lewis 1970 (adding "is next to"). In consequence, mereotopological theories differ widely. But modulo definition or translation, certain basic principles are common to most of them. As an example, consider this list of axioms which are accepted both by Kleinknecht (1992) and Smith (1996); it is formulated in $\mathrm{L}^{1}[\mathrm{o}, \triangleleft]$, where " $\triangleleft$ " is a 2-place predicate read as "is an interior part of": ${ }^{13}$

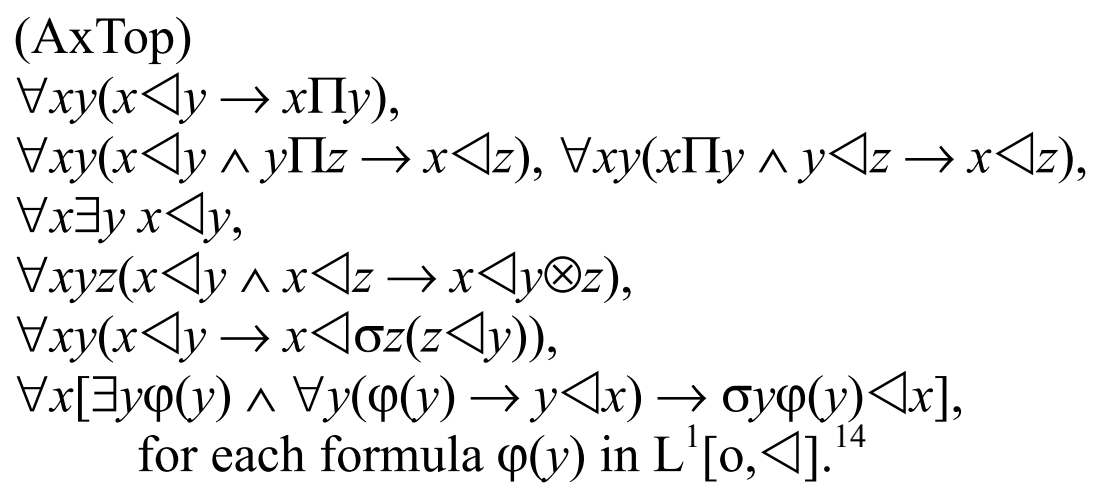

But now, we have the first horn of the WS-dilemma. Let's define

(D2) $x \triangleleft y: \leftrightarrow x \Pi y$.

\section{Lemma 2:}

(i) $\mathrm{CI}+\mathrm{FUS}^{1}(+(\mathrm{D} 2))+($ AxTop).

(ii) If $T$ is a consistent extension of $\mathrm{CI}+\mathrm{FUS}^{1}$ in $\mathrm{L}^{1}[\mathrm{o}]$, then $T+$ (AxTop) is a subtheory of a definitional extension of $T$.

Similar metatheorems can be shown for Clarkes theory and also for $(\mathrm{AxC})$. Thus mereotopological axioms should be added. But which ones? On the one hand, Grzegorczyk (1951) has found a list of sentences in $\mathrm{L}^{1}[\mathrm{o}, C]$ for which he claims that they relatively interpret $\mathrm{Q}$. That's an impressive result. But these sentences can hardly be claimed to be plausible as new mereotopological axioms. Kleinknecht (1992) and Smith (1996), on the other hand, have tried to find such principles: their common idea is to

\footnotetext{
${ }^{13}$ I follow the presentation in Ridder 2002.

${ }^{14}$ I write,$y \otimes z$ " for the common part of $y$ and $z$ (when these overlap) and $\sigma y \varphi(y)$ for the fusion of the $\varphi$ 's.
} 
give boundaries an important status. Kleinknecht defines " $x \mathrm{G} y$ " (for " $x$ is part of a boundary of $y$ ") as " $\forall u(u \triangleleft y \vee u \triangleleft-y \rightarrow \neg u$ o $x) \wedge y \neq 1$ " (Smith's definiens is equivalent for $y \neq 1) .{ }^{15}$ And both accept

$$
\forall y(y \neq 1 \rightarrow \exists z z \mathrm{G} y)
$$

as a theorem of their axiom systems. When it comes to the further axioms, however, a remarkable branching can be found. Whereas

$$
\forall x \exists y \quad y \triangleleft x
$$

is a theorem of Kleinknecht's system $\mathrm{Kl}$,

$$
\forall x(\operatorname{At}(x) \rightarrow \neg \exists y y \triangleleft x)
$$

can be proved in Smith's theory Sm. That is, $\mathrm{Kl}$ is inconsistent with $\mathrm{Sm}$ if the existence of atoms is assumed. Now, who of those two is right intuitively? I must say that I have no strong naive preferences here. But from the point of view of the WS-dilemma, it seems clear to me that $\mathrm{Kl}$ is unacceptable: for $\mathrm{Kl}$ proves that there is exactly one object.

$$
\text { Lemma 3: } \mathrm{K} 1 \vdash \forall x(x=1) \text {. }
$$

This is incompatible with the $2^{\text {nd }}$ horn of the dilemma (if $\mathrm{K} 1$ is a $\mathrm{L}^{1}[0, \triangleleft]$ calculus of individuals).

\subsection{Infinity}

It is sometimes held that our universe W (say, at a specific time), the sum of all concrete things, is finite, or that we should not assume that it is infinite, or that we simply do no know. I think that all these assessments presuppose that "W is (in-) finite" is understood. That may be the case; but it is quite another thing to actually lay down a definition for that phrase. As far as I know, " $x$ is infinite" has only been defined set-or type-theoretically. And prima facie, $\mathrm{W}$ is no set or higher-order object: $\mathrm{W}$ is as concrete as its parts.

\footnotetext{
${ }^{15}$ I write "-y" for the complement of $y$ (when it exists) and " 1 " for that object which has all objects as parts.
} 
Now, to start with, what could a definition of " $x$ is finite" be when we have only $\mathrm{L}^{1}[\mathrm{o}]$ at our disposal? That is, I am searching for a formula $\alpha(x)$ from $\mathrm{L}^{1}[\mathrm{o}]$ which expresses that $\mathrm{x}$ is finite. ${ }^{16} \mathrm{I}$ certainly do not ask whether there is sentence $\varphi$ in $\mathrm{L}^{1}[\mathrm{o}]$ such that for all models $\mathscr{M}$ of $T$ (when $T$ is consistent), $\mathscr{C} \mid=\varphi \Leftrightarrow M$ is finite. For by general model-theoretic reasons (i.e., compactness), there can be none. But this type of result holds for, e.g., ZF, too. And that fact doesn't keep us from believing that there is a set-theoretic formula $\alpha(x)$ which does express that $x$ is finite: for example, " $\exists y(y \in \omega \wedge x$ has the same cardinality as $y)$ " may be chosen for $\alpha(x)$.

I think that when one attempts to determine if there is a similar $\alpha$ in $\mathrm{L}^{1}[\mathrm{o}]$, one first has to lay down axioms for finiteness. In our case, they have to be stated in $\mathrm{L}^{1}[\mathrm{o}, \mathrm{F}]$, i.e., $\mathrm{L}^{1}[\mathrm{o}]$ extended by a new 1-place predicate $\mathrm{F}$ for " $x$ is finite". Here is my suggestion for them:

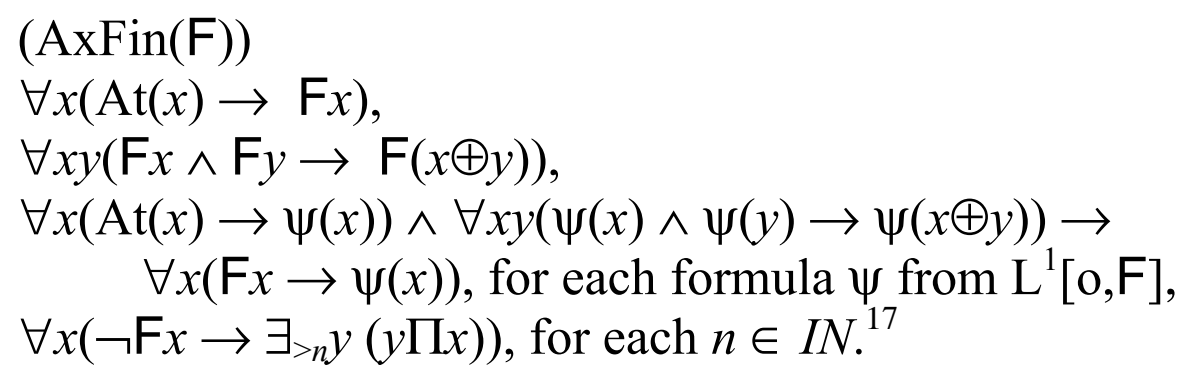

It is unpleasant that the WS-dilemma shows up again; but here it is surprising: " $x$ is finite", as it axiomatized through $(\operatorname{AxFin}(F))$, is definable in $\mathrm{L}^{1}[\mathrm{o}]$. More precisely, define

(D3) $\mathrm{F}(x): \leftrightarrow \forall y(\forall z(\operatorname{At}(z) \rightarrow z \Pi y) \rightarrow x \Pi y)$.

\section{Lemma 4:}

(i) $\mathrm{CI}+\mathrm{FUS}_{\mathrm{AT}}^{1}(+(\mathrm{D} 3))+(\operatorname{AxFin}(\mathrm{F}))$

(ii) If $T$ is a consistent extension of $\mathrm{CI}+\mathrm{FUS}^{1}{ }_{\mathrm{AT}}$ in $\mathrm{L}^{1}[\mathrm{o}]$, then $T+(\operatorname{AxFin}(\mathrm{F}))$ is a subtheory of a definitional extension of $T$.

\footnotetext{
${ }^{16}$ The topic of what it generally means for a formula $\alpha(x)$ to express: $x$ is so-and-so, is a large one. For the special case that $\alpha(x)$ is a set theoretical formula that should express that $x$ is (in-) finite, I have presented a suggestion in Niebergall 2009a. I take this to by typical; but that view is not defended in Niebergall 2009a.

${ }^{17}$ I write " $y \oplus z$ " for the fusion of $y$ and $z$ and " $\exists_{>_{n}} y\left(y \prod x\right)$ " for " $x$ has more than $n$ parts" (cf. section 2.2).
} 
I am nevertheless reluctant to accept that " $\mathrm{F}(x)$ " expresses that $x$ is finite. Here is a reason: it can be shown that $\mathrm{ACI}_{\omega}-\forall x \mathrm{~F}(x)$. Now in each model of $\mathrm{ACI}_{\omega}$, this would mean that its 1 were finite; in fact, however, each has infinitely many parts (naively understood). The most interesting way out of this quandary seems to be to find a better axiomatization: but what could that be?

\section{VARIANTS OF CALCULI OF INDIVIDUALS IN $2^{\mathrm{ND}}$ ORDER LANGUAGES}

\subsection{The general framework}

The simplest way to extend $\mathrm{L}^{1}[\mathrm{o}]$ to a $2^{\text {nd }}$ order language $\mathrm{L}^{2}[\mathrm{o}]$ is to add 1place $2^{\text {nd }}$ order variables ( " $X$ ", $\ldots$ ) to it and define the set of second order terms and formulas by employing these new expressions. Given that, let's look for analogues of the extensions of $\mathrm{CI}$ in $\mathrm{L}^{1}[\mathrm{o}]$. I will call these theories, whether they deserve it or not, "2 $2^{\text {nd }}$ order calculi of individuals". To start with, the $1^{\text {st }}$ order sentences from section 2.1 are a good choice for specific axioms. As regards FUS ${ }^{1}$, one should change it to a $2^{\text {nd }}$ order version. Two amendments are particularly natural:

$\mathrm{FUS}^{2}$, the full $2^{\text {nd }}$ order fusion schema, is the set of all formulas $\mathrm{FUS}_{\varphi}^{2}$, with

$$
\mathrm{FUS}_{\varphi}^{2}:=\lceil\exists x \varphi(x) \rightarrow \exists z \forall y(z \text { o } y \leftrightarrow \exists x(\varphi(x) \wedge x \text { o } y))\rceil,
$$

where $\varphi(x)$ is a formula of $\mathrm{L}^{2}[\mathrm{o}]$ (possibly containing further free variables);

FUS-Ax, the $2^{\text {nd }}$ order fusion axiom:

$$
\forall X(\exists x X x \rightarrow \exists z \forall y(z \text { o } y \leftrightarrow \exists x(X x \wedge x \text { o } y))) .
$$

Further plausible $2^{\text {nd }}$ order principles for "o" are not that easy to find. But there are other $2^{\text {nd }}$ order sentences which usually are taken to be beyond dispute, to be even logical truths. One is a Leibniz principle for identity:

(Leib) $\forall y z(\forall X(X y \leftrightarrow X z) \rightarrow y=z)$.

It will be a consequence of other accepted axioms, however. More interesting and problematic are comprehension schemata. Again, two versions are worthy of special attention: 
Comp ${ }^{2}$, the full $2^{\text {nd }}$ order comprehension schema, is the set of all formulas Comp , with

$\operatorname{Comp}_{\varphi}:=\lceil\exists X \forall x(X x \leftrightarrow \varphi(x))\rceil$,

where $\varphi(x)$ is a formula of $\mathrm{L}^{2}[\mathrm{o}]$ (possibly containing further free variables);

Comp ${ }^{1}$, the restricted $2^{\text {nd }}$ order comprehension schema, is the set of all formulas $\mathrm{Comp}_{\varphi}$, where $\varphi$ is a formula of $\mathrm{L}^{2}[\mathrm{o}]$ containing no bound $2^{\text {nd }}$ order variables.

On first sight, we therefore have four $2^{\text {nd }}$ order variants of one $1^{\text {st }}$ order calculus of individuals $T: T \cup\left\{\right.$ FUS-Ax $\cup$ Comp $^{1}, T \cup\{$ FUS-Ax $\}$ $\cup \mathrm{Comp}^{2}, T \cup \mathrm{FUS}^{2} \cup \mathrm{Comp}^{1}, T \cup \mathrm{FUS}^{2} \cup \mathrm{Comp}^{2}$. Normally, we are not interested in sets of axioms, however, but in the theories induced by them. And this is the place where one has to be careful when dealing with $2^{\text {nd }}$ order languages instead of $1^{\text {st }}$ order ones. For there are many non-equivalent ways to define logical consequence for $2^{\text {nd }}$ order languages. Two types of them are employed in practice. Thus, we usually have two serious candidates for the theory $T$ given by one set of $2^{\text {nd }}$ order sentences $\Sigma$.

Let me recall the two definitions of " $\varphi$ follows from $\Sigma$ ", where $\Sigma$ is a set of sentences from $\mathrm{L}^{2}[\mathrm{o}]$ and $\varphi$ is a sentence from $\mathrm{L}^{2}[\mathrm{o}]$.

The structures which are appropriate for evaluating expressions from $\mathrm{L}^{2}[\mathrm{o}]$ are now taken to be of the form $\left\langle M, \Omega, \mathrm{o}^{M}\right\rangle$, in short $\mathscr{L}$. If $\Omega$ is a nonempty subset of $\wp(M), \mathscr{C}^{2}$ is called a generalized $2^{\text {nd }}$ order structure (in short: g2-structure). In the special case that $\Omega=\wp(M)$, it is called a standard $2^{\text {nd }}$ order structure (in short: s2-structure).

Let $\mathscr{L}^{2}$ be a g2-structure. A variable assignment $\beta$ (relative to $\mathscr{A}^{2}$ ) is a function defined on the set of $1^{\text {st }}$ and $2^{\text {nd }}$ order variables which maps the $1^{\text {st }}$ order variables to elements of $M$ and the $2^{\text {nd }}$ order variable to elements of $\Omega$. Given all that, evaluation in a structure is defined as it is known from the $1^{\text {st }}$ order case, with one interesting clause added (for a formula $\varphi$ of $\mathrm{L}^{2}[\mathrm{o}] ; \beta(\mathrm{X}: \mathrm{C})$ is the variant of $\beta$ which differs from $\beta$ at most in mapping $X$ to $C$ ): 


$$
\mathscr{C}^{2}, \beta \mid=\forall \mathrm{X} \varphi \Leftrightarrow \forall \mathrm{C}\left(\mathrm{C} \in \Omega \Rightarrow \mathscr{C}^{2}, \beta(\mathrm{X}: \mathrm{C}) \mid=\varphi\right){ }^{18}
$$

Now the common metalogical vocabulary, such as "satisfiability", "logical truth" and "logical consequence", can be defined as usual, via g2structures. But there is also the alternative of taking only s2-structures into account. Thus, let's consider logical consequence (if $\Sigma$ is a set of sentences from $\mathrm{L}^{2}[\mathrm{o}]$ and $\varphi$ is a sentence from $\left.\mathrm{L}^{2}[\mathrm{o}]\right)$ :

$$
\begin{aligned}
\Sigma \mid={ }^{\mathrm{g} 2} \varphi: \Leftrightarrow & \forall M \Omega \mathrm{o}^{M}\left(\left\langle M, \Omega, \mathrm{o}^{M}\right\rangle \text { is a g2-structure } \Rightarrow\right. \\
& \left.\left(\left\langle M, \Omega, \mathrm{o}^{M}\right\rangle\left|=\Sigma \Rightarrow\left\langle M, \Omega, \mathrm{o}^{M}\right\rangle\right|=\varphi\right)\right), \\
\Sigma \mid={ }^{\mathrm{s} 2} \varphi: \Leftrightarrow & \forall M \Omega \mathrm{o}^{M}\left(\left\langle M, \Omega, \mathrm{o}^{M}\right\rangle \text { is a s2-structure } \Rightarrow\right. \\
& \left.\left(\left\langle M, \Omega, \mathrm{o}^{M}\right\rangle\left|=\Sigma \Rightarrow\left\langle M, \Omega, \mathrm{o}^{M}\right\rangle\right|=\varphi\right)\right) .
\end{aligned}
$$

Since each s2-structure is a g2-structure, we immediately get: $\Sigma\left|={ }^{\mathrm{g} 2} \varphi \Rightarrow \Sigma\right|={ }^{\mathrm{s} 2} \varphi$. But for $2^{\text {nd }}$ order languages in general, the converse is far from true. In addition, the notorious incompleteness and categoricity metatheorems which are so often attributed to $2^{\text {nd }}$ order "logic" and to certain $2^{\text {nd }}$ order theories are only true under the presupposition that the $2^{\text {nd }}$ order languages for which they are stated are interpreted employing only s2-structures. If all of the g2-structures are admitted as models instead, these "metatheorems" are false: in this case, we have a completeness and compactness theorem for $2^{\text {nd }}$ order consequence and versions of the Löwenheim-Skolem theorems (see Leivant 1994 for more on this).

\subsection{Some metatheorems}

With the distinctions just sketched as a background, let me introduce some abbreviations. In what follows, let $\Sigma$ be a set of $\mathrm{L}^{2}[\mathrm{o}]$-sentences.

$$
\begin{aligned}
& \Sigma^{\mathrm{g} 2+}:=\left\{\varphi \mid \varphi \text { is a } \mathrm{L}^{2}[\mathrm{o}] \text {-sentence } \wedge \Sigma \cup \mathrm{CI} \cup \mathrm{FUS}^{2} \cup \operatorname{Comp}^{2} \mid={ }^{\mathrm{g} 2} \varphi\right\}, \\
& \Sigma^{\mathrm{g} 2-}:=\left\{\varphi \mid \varphi \text { is a L }{ }^{2}[\mathrm{o}] \text {-sentence } \wedge \Sigma \cup \mathrm{CI} \cup\{\text { FUS-Ax }\} \cup \text { Comp }^{1} \mid={ }^{\mathrm{g} 2} \varphi\right\} \text {, } \\
& \Sigma^{\mathrm{g} 2}:=\left\{\varphi \mid \varphi \text { is a L }{ }^{2}[\mathrm{o}] \text {-sentence } \wedge \Sigma \cup \mathrm{CI} \cup \mathrm{FUS}^{2} \cup \operatorname{Comp}^{1} \mid={ }^{\mathrm{g} 2} \varphi\right\} \text {, } \\
& \Sigma^{\mathrm{s} 2}:=\left\{\varphi \mid \varphi \text { is a } \mathrm{L}^{2}[\mathrm{o}] \text {-sentence } \wedge \Sigma \cup \mathrm{CI} \cup \mathrm{FUS}^{2} \cup \operatorname{Comp}^{2} \mid={ }^{\mathrm{s} 2} \varphi\right\} \text {. }
\end{aligned}
$$

18 s2-structures in particular may alternatively be construed as being the same objects as $1^{\text {st }}$ order structures, i.e., ordered pairs $\left\langle M, o^{M}\right\rangle$. Satisfaction in such structures is for $2^{\text {nd }}$ order quantifiers explained as follows:

$$
\left\langle M, \mathrm{o}^{M}\right\rangle, \beta \mid=\forall \mathrm{X} \varphi \Leftrightarrow \forall \mathrm{C}\left(\mathrm{C} \subseteq M \Rightarrow\left\langle M, \mathrm{o}^{M}\right\rangle, \beta(\mathrm{X}: \mathrm{C}) \mid=\varphi\right) .
$$




\section{Lemma 5:}

(i) $\Sigma^{\mathrm{g} 2-} \subseteq \Sigma^{\mathrm{g} 2} \subseteq \Sigma^{\mathrm{g} 2+} \subseteq \Sigma^{\mathrm{s} 2}$.

(ii) If $\Sigma$ is a set of $\mathrm{L}^{1}[\mathrm{o}]$-sentences, then $\Sigma+\mathrm{CI}+\mathrm{FUS}^{1} \subseteq \Sigma^{\mathrm{g} 2-}$.

(iii) If $\mathscr{A}^{2}$ is a s2-structure, then $\mathscr{A}^{2} \mid=\mathrm{Comp}^{2}$.

(iv) $\Sigma^{\mathrm{s} 2}=\left\{\varphi \mid \varphi\right.$ is a $\mathrm{L}^{2}[\mathrm{o}]$-sentence $\wedge \Sigma \cup \mathrm{CI} \cup\{$ FUS-Ax $\left.\} \mid={ }^{\mathrm{s} 2} \varphi\right\}$.

The correspondence between mereological and Boolean algebras and the translation from $\mathrm{L}^{1}[\mathrm{o}]$ to $\mathrm{L}^{1}[\mathrm{BA}]$ can be extended to the $2^{\text {nd }}$ order counterparts of the structures and of the languages. Let me mention merely some of the consequences of this approach:

Lemma 6: If $\left\langle B, \Pi^{B}, \sqcup^{B},-^{B}, 0^{B}, 1^{B}\right\rangle$ is a complete Boolean algebra, then $\left\langle B^{-}, \wp\left(B^{-}\right), \mathrm{o}^{B-}\right\rangle \mid=$ FUS-Ax.

Now the Boolean algebras from section 2.3 are complete (i.e. have suprema for each nonempty subset of their domain). Therefore, they induce s2-structures which are models of the $\left(\mathrm{ACI}_{n+1}\right)^{\mathrm{s} 2}$ and $\left(\mathrm{MCI}_{n+1}\right)^{\mathrm{s} 2}$, or of $\left(\mathrm{ACI}_{\omega}\right)^{\mathrm{s} 2},(\mathrm{FCI})^{\mathrm{s} 2}$ or $\left(\mathrm{MCI}_{\omega}\right)^{\mathrm{s} 2}$, respectively.

We also have sort of a converse to Lemma 6:

Lemma 7: For each $\mathscr{A}^{2}\left(=\left\langle M, \wp(M), \mathrm{o}^{M}\right\rangle\right)$ which is a s2-structure satisfying CI, FUS-Ax, there is a complete Boolean algebra $\mathscr{B}$ such that $\mathscr{B}=\left\langle M, \mathrm{o}^{M}\right\rangle$.

In the $1^{\text {st }}$ order case, there is a similar, if weaker, metatheorem:

Lemma 8: For each mereological algebra $\mathscr{C}$ which satisfies $\mathrm{FUS}^{1}{ }_{\mathrm{AT}}$, there is a complete Boolean algebra $\mathscr{B}$ such that $\mathscr{B} \equiv \mathscr{M}$.

Lemma 8 can nonetheless be employed to obtain a rather strong conservativity result:

\section{Theorem 6:}

(i) If $\Sigma$ is a set of $\mathrm{L}^{1}[\mathrm{o}]$-sentences, then $\Sigma+\mathrm{CI}+\mathrm{FUS}^{1} \mid \mathrm{L}^{1}[\mathrm{o}]=$ $\Sigma^{\mathrm{s} 2} \mid \mathrm{L}^{1}[\mathrm{o}]$.

(ii) If $\Sigma$ is a set of $\mathrm{L}^{1}[\mathrm{o}]$-sentences, then $\Sigma^{\mathrm{g} 2-}, \Sigma^{\mathrm{g} 2}, \Sigma^{\mathrm{g} 2+}$ and $\Sigma^{\mathrm{s} 2}$ contain the same sentences from $\mathrm{L}^{1}[\mathrm{o}]$. 
By the above observations, all of the $2^{\text {nd }}$ order variants of the $1^{\text {st }}$ order theories $\mathrm{ACI}_{\mathrm{n}+1}$ and $\mathrm{MCI}_{\mathrm{n}+1}, \mathrm{ACI}_{\omega}, \mathrm{FCI}$ and $\mathrm{MCI}_{\omega}+\mathrm{FUS}_{\mathrm{AT}}^{1}$ are consistent and not distinguishable by their $1^{\text {st }}$ order consequences. But are they maximal consistent? And what is their relation as to arbitrary sentences from $\mathrm{L}^{2}[\mathrm{o}]$ ? A lot of research has still to be done here. Let me close this paper with the nontrivial example of $2^{\text {nd }}$ order extensions of $\mathrm{ACI}_{\omega}$.

\section{Lemma 9:}

(i) $\quad\left(\mathrm{ACI}_{\omega}\right)^{\mathrm{s} 2}$ is not maximal consistent.

(ii) All s2-structures of cardinality $2^{\text {so }}$ which are models of $\left(\mathrm{ACI}_{\omega}\right)^{\mathrm{s} 2}$ are isomorphic with each other.

(iii) There is a sentence "CounAt"19 from $\mathrm{L}^{2}[\mathrm{o}]$ such that for all s2structures $\left\langle M, \wp(M), \mathrm{o}^{M}\right\rangle$ which are models of $\left(\mathrm{ACI}_{\omega}\right)^{\mathrm{s} 2}$, $\left\langle M, \wp(M), \mathrm{o}^{M}\right\rangle \mid=$ CountAt $\Leftrightarrow\left\{a \mid a\right.$ is an atom $\left.{ }^{M}\right\}$ is countably infinite.

\section{Theorem 7:}

(i) All s2-structures which are models of $\left(\mathrm{ACI}_{\omega}+\mathrm{CounAt}\right)^{\mathrm{s} 2}$ are isomorphic with each other.

(ii) $\left(\mathrm{ACI}_{\omega}+\mathrm{CounAt}\right)^{\mathrm{s} 2}$ is maximal consistent.

In distinction, $\left(\mathrm{ACI}_{\omega}+\mathrm{CounAt}\right)^{\mathrm{g} 2+}$ is a proper subtheory of $\left(\mathrm{ACI}_{\omega}+\right.$ CounAt $)^{\mathrm{s} 2}$ and therefore fails to be maximal consistent.

\section{REFERENCES}

BREITKOPF 1978: A. Breitkopf, Axiomatisierung einiger Begriffe aus Nelson Goodmans The Structure of Appearance, in: Erkenntnis 12 (1978), pp.229-247.

Clarke 1981: B. Clarke, A Calculus of Individuals Based on 'Connection', in: Notre Dame Journal of Formal Logic 22 (1981), pp.204-218.

Clarke 1985: B. Clarke, Individuals and points, in: Notre Dame Journal of Formal Logic 26 (1985), pp.61-75.

EBERLE 1970: R. Eberle, Nominalistic Systems, Dordrecht: Reidel.

FEFERMAN 1960: S. Feferman, Arithmetization of Metamathematics in a General Setting, in: Fundamenta Mathematicae 49 (1960), pp.35-92.

FIELD 1980: H. Field, Science without Numbers: A Defence of Nominalism, Oxford: Blackwell.

GoODmAN 1951: N. Goodman, The Structure of Appearance, Dordrecht: Reidel.

\footnotetext{
${ }^{19}$ This sentence is taken from Lewis 1991.
} 
Goodman 1958: N. Goodman, On Relations that Generate, in: Philosophical Studies 9 (1958), pp.65-66; reprinted in N. Goodman, Problems and Projects, Indianapolis: Bobbs-Merril. pp.171-172.

GRZEGORCZYK 1951: A. Grzegorczyk, Undecidability of some topological theories, in: Fundamenta Mathematicae 38 (1951), pp.137-152.

Halmos 1963: P. Halmos, Lectures on Boolean Algebras. Princeton, NJ: Van Nostrand.

HELLMAN 1969: G. Hellman, Finitude, infinitude, and isomorphism of interpretations in some nominalistic calculi, in: Nô̂s 3 (1969), pp.413-425.

HENDRY 1982: H.E. Hendry, Complete Extensions of the Calculus of Individuals, in: Noûs 16, pp.453-460.

Hodges/LewIS 1968: W. Hodges/D. Lewis, Finitude and infinitude in the atomic calculus of individuals, in: Noûs 2 (1968), pp.405-410.

KLEINKNECHT 1992: R. Kleinknecht, Mereologische Strukturen der Welt, in: Wissenschaftliche Zeitschrift der Humboldt-Universität zu Berlin, Reihe Geistes- und Sozialwissenschaften 41 (1992), pp.40-53.

LeIVANT 1994: D. Leivant, Higher Order Logic, in D. Gabbay, C.J. Hogger, J.A. Robinson (ed.): Handbook of Logic in Artificial Intelligence and Logic Programming, Volume 2, Clarendon Press, pp.229-321.

LEONARD/GOODMAN 1940: H. Leonard/N. Goodman, The Calculus of Individuals and its Uses, in: The Journal of Symbolic Logic 5 (1940), pp.45-55.

LEWIS 1970: D. Lewis, Nominalistic Set Theory, in: Nô̂s 4 (1970), pp.225-240.

LEWIS 1991: D. Lewis, Parts of Classes, Oxford: Blackwell.

NiEBERGALL 2000: K.G. Niebergall, On the Logic of Reducibility: Axioms and Examples, in: Erkenntnis 53 (2000), pp.27-61.

NiEBERGALL 2005: K.G. Niebergall, Zur nominalistischen Behandlung der Mathematik, in J. Steinbrenner, O. Scholz und G. Ernst (Hrsg.): Symbole, Systeme, Welten: Studien zur Philosophie Nelson Goodmans, Heidelberg: Synchron Wissenschaftsverlag der Autoren, pp.235-260.

NIEBERGALL 2007: K.G. Niebergall, Zur logischen Stärke von Individuenkalkülen, in: H. Bohse und S. Walter (Hgg.); Ausgewählte Sektionsbeiträge der GAP.6, Sechster Internationaler Kongress der Gesellschaft für Analytische Philosophie, Berlin, 11.-14. September 2006. (CD-ROM) Paderborn: mentis.

NiEBERGALL 2009A: K.G. Niebergall, Unendlichkeit ausdrücken und Unendlichkeitsannahmen machen, to appear in: Deutsches Jahrbuch Philosophie 3.

NiEBERGALL 2009B: K.G. Niebergall, Zur Metatheorie mereologischer Theorien, manuscript, Berlin 2009.

RIDDER 2002: L. Ridder, Mereologie. Ein Beitrag zur Ontologie und Erkenntnistheorie. Frankfurt a.M.: Klostermann.

SMITH 1996: B. Smith, Mereotopology: a theory of parts and boundaries, in Data and Knowledge Engineering 20 (1996), pp.287-303.

TARSki/Mostowski/Robinson 1953: A. Tarski/A. Mostowski/R.M. Robinson, Undecidable theories, Amsterdam: North-Holland.

Yoes 1967: M.G. Yoes, Jr., Nominalism and non-atomic systems, in: Nô̂s 1 (1967), pp.193-200. 